Research Article

\title{
Integrated Medicine Strongly Improves Post-Stroke Rehabilitative Performance in a Public Health Facility in Italy
}

Simonetta Bernardini ${ }^{1,}{ }^{*}$, Francesca Bechini ${ }^{2}$, Giulia Braccesi ${ }^{1}$, Franco Cracolici ${ }^{1}$, Rosaria Ferreri ${ }^{1}$, Roberto Pulcri ${ }^{1}$, Andrea Serafini ${ }^{3}$

1. Department of Integrated Medicine, Tuscany Reference Centre for Integrated Medicine in the Hospital Pathway, Pitigliano Hospital, Italy; E-Mails: bernardini@sbernardini.it; giulia.braccesi@gmail.com; francocracolici@yahoo.it; tyvvf@tin.it; robertopulcri @gmail.com

2. Junior doctor at Tuscany Reference Centre for Integrated Medicine in the Hospital Pathway, Pitigliano Hospital, Italy; E-Mail: bechins@outlook.it

3. Medical management, Misericordia Hospital, Grosseto; E-Mail: andrea.serafini@uslsudest.toscana.it

* Correspondence: Simonetta Bernardini; E-Mail: bernardini@s-bernardini.it

Academic Editors: Elio Rossi, Sonia Baccetti and Mariella Di Stefano

Special Issue: Experience and activities of the Regional Tuscan Centre for Integrative Medicine

OBM Integrative and Complementary Medicine 2019, volume 4, issue 2

doi:10.21926/obm.icm.1902024
Received: September 30, 2018

Accepted: April 11, 2019

Published: April 17, 2019

\begin{abstract}
Since 2011, the Tuscany Regional Authority has been funding the use of Integrated Medicine (IM) in a clinical setting in Pitigliano Hospital, with the aim of testing the true efficacy of complementary and alternative medicine (CAM) in a hospital setting. The clinical setting also comprises the Manciano rehabilitation facility whose services include post-stroke rehabilitation.

This study aims to establish if IM helps to improve the rehabilitation performance of stroke patients. The study compared 212 patients divided into two groups: 94 treated with IM (conventional therapy plus homeopathy and acupuncture) and 118 treated with conventional therapy alone. All patients were evaluated with the rehabilitation scores in use
\end{abstract}

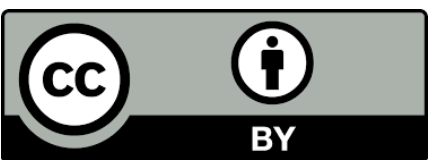

(C) 2019 by the author. This is an open access article distributed under the conditions of the Creative Commons by Attribution License, which permits unrestricted use, distribution, and reproduction in any medium or format, provided the original work is correctly cited. 
at the center (Trunk Control Test and Barthel Index) before and after completing the rehabilitation course. The results demonstrate a considerable benefit for the use of IM with a significance of $p=0.001$ for the Trunk Control Test difference and $p<0.001$ for the Barthel Index difference.

No side effects were documented in any patient treated with IM. The level acceptance of the integrated therapies was high (accepted by $95.94 \%$ of patients: 94 out of 98 inpatients).

\section{Keywords}

Orthodox medicine; integrated medicine; acupuncture; homeopathy; post-stroke rehabilitation performance

\section{Introduction}

Cerebral stroke is the second cause of death, the third cause of disability worldwide and the first cause of disability in the elderly. Taken as a whole, $35 \%$ of individuals suffering a stroke will have a persistent severe disability. Age is the main risk factor [1]: $75 \%$ of cases occur in patients aged 65 years or older. In Europe, Italy has the highest percentage of people aged over 65 (19.9\%), and the number of new cases of stroke is forecast to reach 195,000 per year in 2020 [2]. According to the World Health Organization, the number of strokes in Europe is destined to rise from 1.1 million per year in 2000 to more than 1.5 million per year in 2025 [3].

Although there is a high mortality rate in the first month after the acute event, an estimated $35 \%$ of patients survive more than 10 years. Functional and neurological recovery is the main challenge facing stroke patients. One month after the event, $55 \%$ of patients have lost their previous lifestyle and are dependent on others for activities of daily living. Numerous studies agree that the greatest functional improvement can be achieved in the first phase (1-3 months) after the stroke, even though recovery can still continue thereafter [4]. In any case, 35-40\% of survivors will continue to suffer a severe disability while a high percentage will have milder impairments affecting mobility, more complex activities of daily living and quality of life, despite the progress achieved in neurological rehabilitation in recent decades $[5,6]$. Given this situation, it is particularly useful to consider, in a setting of Integrated Medicine (IM), any complementary or alternative medicine (CAM) which might improve the recovery of stroke patients.

As already reported [7, 8], an IM Hospital was opened in Tuscany in February 2011 which has now become the Tuscany Reference Centre for Integrated Medicine in Hospitals. The center forms part of the regional public health service and now has three active clinical settings involving IM: one in Pitigliano Hospital, one in the Manciano Rehabilitation Center and one in the largest hospital in its province, Grosseto. The Manciano center's patients [7] include stroke patients, who convalesce in the center for three weeks after discharging from acute care to undergoing a rehabilitation programme involving physiotherapists, neurologists and orthopaedists. Since February 2011, these inpatients have also been offered CAM (homeopathy and acupuncture). The sole enrollment criterion is their presence at the center following their admission on Wednesday or Thursday, the days when the CAM doctors attend the center. In any case, the opportunity to enroll them is the result of an objective shared by both the conventional doctors and CAM experts 
making use of the clinical setting in place at the center [7]. Almost all of the invited patients (95.92\%) agreed to the integrated therapy and signed an integrated therapy consent form.

\section{Materials and Methods}

\subsection{Patient Selection and Inclusion Criteria}

All patients admitted from 2013 to 2017 in the rehabilitation clinic of Manciano who suffered a stroke (ischemia or hemorrhagic) were enrolled in the study.

The enrolling criteria in the IM protocol was random: to all of the patients admitted on Wednesday and Thursday, the IM treatment was proposed (95.94\% of agreement); to all of patients admitted on Monday or Tuesday, the standard protocol was administered.

Between 2013 and 2017, 212 patients were included in the study: 94 patients treated with conventional medicine plus IM were compared against 118 patients treated with conventional methods alone. The variables considered were the Barthel Index (BI) and the Trunk Control Test (TCT) [8].

\subsection{Data Managing}

For this study, we set the outcome variables as the Trunk control (TC) difference and Barthel (B) difference between admission and discharge value. The independent variables were the treatment (whereas treated with the homeopathic and acupuncture protocol or with the standard protocol), age, sex, and diagnosis (whereas ischemic or hemorrhagic stroke).

\subsection{Statistical Analysis}

For both groups (treated with $\mathrm{MI}$ and with standard protocol), we calculated the mean of age, Barthel index admission, Trunk Control test admission, male to female and ischemia to hemorrhagic stroke ratio. A statistical test (Mann Whitney test for continuous variables and $\chi^{2}$ test for categorical ones) was carried on to verify possible differences between the groups. To investigate possible correlations between outcome and independent variables, we performed a bivariate analysis for each outcome. For continuous independent variables (age), we used the Spearman test, while for categorical ones (treatment, sex, diagnosis) we used the Mann-Whitney test.

All patients stayed in the facility for three weeks. The IM patients received two acupuncture sessions a week and homeopathic medicines daily, as prescribed by the homeopathic doctors and prepared by the hospital pharmacy's galenics department. All IM patients were also treated with the routine conventional therapy in use at the rehabilitation center.

The adverse effects of homeopathy and acupuncture have been monitored over the years.

Moreover, the homeopathic therapy cost has been calculated.

The data used to support the findings of this study were provided by ASL Sudest, Tuscany under license, and so cannot be made freely available. Access to these data will be considered by the authors upon request, with the permission of ASL Sudest, Tuscany. 


\section{IM Protocols Used}

\subsection{Homeopathy}

Magistral preparations. The active substance was chosen on the basis of homeopathic similarity to actual symptoms, the "reactive model" of the disease and the homeopathic constitution of the patient. The following homeopathic medicines were used in all cases: Lachesis m.; Plumbum m.; Gelsemium s.; and Arnica m. at a $30 \mathrm{C}$ dilution. A constitutional homeopathic medicine, selected on the basis of the features emerging from the homeopathic interview with each individual patient, was then added to the formulation. The decision to use a magistral formulation was based on its practical advantages: better patient compliance, especially in the case of elderly patients and those not accustomed to taking homeopathic medicines, reproducibility of the treatment protocol, and contained cost.

\subsection{Acupuncture}

The applied protocol focuses on the improvement of aphasia, dysphagia and hemiparesis, frequently associated with hemispatial neglect. We therefore applied a combination of classic points recognized internationally as follows:

Aphasia CV 23 LU 7 PC 6 KI 6 HT 5 GV 16, which we matched with Yamamoto's neurological technique, adding a personal Shen point which interfaces with emotive suffering.

Dysphagia: TR 23 GV 20 GV 26 GB 2; here too, a combination of Yamamoto's complex technique was used with Yamamoto's Area A1-A8 and functional ypsilon points, Broca's area and Yamamoto's modified Wernicke's area, and areas connected to the parietal sutures and the eyebrow areas, also using Su-Jok and finally, stimulating the mouth auricular point.

Hemiparesis: classic treatment with ipsilateral LI $16 \mathrm{LI} 11 \mathrm{LI} 4$ at the top and ipsilateral GB 34 ST 36 ST 41 LR 3 at the bottom, to which we added contralaterally the Yamamoto points found in the temporoparietal area and the cerebrum and cerebellum points below the hairline, with electrostimulation mainly at multiple frequencies of $8 \mathrm{~Hz}$.

We personalized the treatments through the emotive points from CV 19 to CV 12. Abdominal acupuncture and navel acupuncture were applied to the most resistant cases.

To assess the effect of the treatment adjusted for age, sex and diagnosis, we performed two multiple regression models (one for each outcome).

\section{Results}

In Table 1, there is the comparison between the control and treated group. There was no significant differences between the groups for sex, diagnosis, Barthel and Trunk control values on admission; the patients in the treated group were significantly younger (6.4 years) than the controls, which means that, except for the age, the groups were similar and without significant differences.

In the bivariate analysis (Table 2), we investigated the relationship between the outcomes (Trunk control and Barthel difference) and the independent variables: for both outcomes, treatment was the only significant variable, with greater differences between admission and discharge in the treated group: 16.7 in IM vs 11.2 in traditional for Trunk control difference, and 
21.6 in IM vs 14.7 in traditional for Barthel difference, which seem to be an huge difference. Sex, diagnosis and age does not seem to affect significantly the outcomes.

Table 1 Stroke patients, comparison of variables with $(n=94)$ and without $(n=118)$ Integrated Medicine (IM).

\begin{tabular}{llll}
\hline Variables & Treated $\mathbf{n = 9 4}$ & Controls $\mathbf{n = 1 1 8}$ & $\mathbf{p}$ \\
\hline Mean age & 68.0 & 74.4 & 0.01 \\
Male \% & $73.4 \%$ & $62.7 \%$ & 0.19 \\
Ischaemic stroke \% & $76.6 \%$ & $74.6 \%$ & 0.45 \\
Barthel index admission mean & 54.31 & 50.41 & 0.73 \\
Trunk control test admission mean & 57.73 & 60.47 & 0.24 \\
\hline
\end{tabular}

Table 2 Bivariate analysis.

\begin{tabular}{lllll}
\hline Outcome & Variable & & value & $\mathbf{p}$ \\
\hline Trunk control & treatment & Mean IM & 16.7 & 0.01 \\
difference & & Mean controls & 11.2 & \\
& sex & Mean male & 12.3 & 0.13 \\
& \multirow{2}{*}{ diagnosis } & Mean female & 16.2 & \\
& & Mean ischaemic & 12.6 & 0.12 \\
& age & coefficient & 16.8 & \\
\hline Barthel difference & treatment & Mean IM & 0.19 & 0.19 \\
& & Mean controls & 21.6 & $<0.001$ \\
& \multirow{2}{*}{ sex } & Mean male & 14.7 & \\
& & Mean female & 19.3 & 0.22 \\
& diagnosis & Mean ischaemic & 17.7 & 0.74 \\
& & Mean haemorrhagic & 17.9 & \\
& & coefficient & 0.04 & 0.89 \\
\hline
\end{tabular}

Treatment (Integrated Medicine: IM) is the most significant variable which positively affects Trunk control and Barthel differences, independently by age, sex and diagnosis.

However, we have seen in Table 1 that the IM group was significantly younger than the traditional one, and because age could be an important variable in term of rehabilitation efficacy, in order to adjust the effect of the treatment mainly for age, but also for sex and diagnosis, we performed two different multiple regression model (one for each outcome). From Table 3 and Table 4 it is possible to assess that treatment is the most significant variable, which positively 
affects Trunk control and Barthel differences, independently by age, sex and diagnosis. Trunk control difference is influenced by sex too: males have smaller differences than females.

Regarding the adverse events: no adverse events were documented with homeopathic magistral preparations; minor adverse events were reported with acupuncture treatments, such as transient bleeding, mild hematoma, and slight pain at the acupoint. To conclude, no serious adverse events have been reported.

Table 3 Multiple regression model for Trunk control difference.

\begin{tabular}{llllll}
\hline \multicolumn{2}{l}{ Trunk control difference } & \multicolumn{5}{l}{ Confidence Interval } \\
\hline Variable & coefficient & Std. Err & $\mathbf{t}$ & $\mathbf{p}$ & \multicolumn{1}{l}{ t } \\
\hline treatment & 9.32 & 2.49 & 3.74 & $<0.001$ & $2.85-11.83$ \\
sex & -5.67 & 2.48 & -2.28 & 0.02 & $-10.58--0.76$ \\
diagnosis & -5.33 & 0.20 & 1.50 & 0.06 & $-10.88-0.22$ \\
age & 0.30 & 2.81 & -1.90 & 0.13 & $-0.09-0.69$ \\
\hline
\end{tabular}

Table 4 Multiple regression model for Barthel difference.

\begin{tabular}{llllll}
\hline \multicolumn{2}{l}{ Barthel difference } & \multicolumn{3}{l}{} \\
\hline Variable & coefficient & Std. Err & $\mathbf{t}$ & $\mathbf{p}$ & Confidence Interval \\
\hline treatment & 10.48 & 2.76 & 3.80 & $<0.001$ & $5.04-15.93$ \\
sex & -2.04 & 2.75 & -0.75 & 0.46 & $-7.47-3.38$ \\
diagnosis & 0.15 & 3.10 & 0.05 & 0.96 & $-5.98-6.27$ \\
age & 0.18 & 0.22 & 0.83 & 0.41 & $-0.25-0.35$ \\
\hline
\end{tabular}

In this study, we compared two groups of patients with similar criteria (type of stroke: ischemic or hemorrhagic), BI and TCT scores on admission and discharge, age and male to female ratio). The only variable which differed significantly was age (Table 1), with the IM treatment group younger (mean age 67.96 years) than the control group (mean age 74.36 years); the youngest and oldest patients in the IM group were aged 55 and 89 years, compared with 60 and 92 years in the control group.

In the bivariate analysis (Table 2) and the two multiple regression tests (Table 3 and Table 4), the only significant variable affecting the BI and TCT outcomes was the treatment type, while the other variables (including the age difference) did not significantly affect the outcomes.

The homeopathic therapy cost, calculated by the galenic laboratory of the Hospital, is about $7 €$ per week.

\section{Discussion}

Given the major persistent disability caused by stroke, it is understandable that post-stroke therapy should also consider the therapeutic resources offered by IM. In the United States, $46 \%$ of stroke survivors use one or more forms of CAM; this figure rises to $54 \%$ in the Far East (Korea) [9]. 
Acupuncture [9-11] is the most commonly used form of CAM in stroke patients. In Canada, a survey of stroke survivors found that $98 \%$ of those interviewed would like more information on the use of acupuncture in post-stroke rehabilitation, and $87 \%$ would be willing to consider acupuncture as a therapeutic option [12]. Numerous reviews and animal studies [13] agree on the utility of acupuncture in post-stroke rehabilitation therapy for the treatment of numerous sequela [14-17]. It has also been demonstrated as a valid tool for the treatment of post-stroke shoulder pain [18], aphasia [19], and dysphagia [20]. However, there is little information on the use of homeopathy in stroke. An interesting randomized controlled trial actually investigated the posttraumatic brain damage [21], while other studies produced inconclusive data [22, 23].

To our knowledge, there is a complete lack of studies of IM, which combine homeopathy with acupuncture in stroke patients. The protocol described herein was made possible by the opportunity offered by the Tuscany Regional Authority to open an IM center under the umbrella of the regional health service, now active since 2011. The setting at the Manciano center proved highly effective from the very beginning $[7,8]$ as no conflicts between the CAM doctors, conventional doctors (neurologists and orthopaedists) and other medical staff (physiotherapists, speech therapists and nurses) have ever arisen. We believe that the small size of the facility, which has just 21 beds, was a great help in fostering effective and harmonious cooperation between the various health workers and the patients. We also believe that the length of time the patients can stay in the facility is key to this cooperation, as they have already completed their urgent and/or emergency medical care elsewhere and come to the center for their rehabilitation program. It is this time which enables the benefits offered by $\mathrm{IM}$ to be verified by comparison against patients who have not undergone the integrated therapeutic protocol.

All of the patients stayed in the facility for three weeks. Those making use of the IM service undergo approximately six acupuncture sessions (twice a week) and daily homeopathy therapy. It is reasonable to think that the opportunity to undergo an acupuncture session every day [24] would further improve the patients' rehabilitation performance. The Manciano center is unable to provide such a service, as the IM team has to work at three different sites, which are not in close proximity and are reachable only by car. In any case, based on our experience, we believe that homeopathy can make up for the reduced number of acupuncture sessions through its daily activation of a different way of stimulating the body towards self-recovery.

Regardless of the conditions which could favour the establishment of an IM setting, the fact remains that the results it produces are noteworthy and, in our opinion, merit attention. In this study, we compared two groups of patients with similar criteria (type of stroke: ischemic or hemorrhagic), $\mathrm{BI}$ and TCT scores on admission and discharge, age and male to female ratio). The only variable which differed significantly was age (Table 1), with the IM treatment group younger (mean age 67.96 years) than the control group (mean age 74.36 years). It can be seen that all of our patients are rather elderly, in any case.

In the bivariate analysis (Table 2) and the two multiple regression tests (Table 3 and Table 4), the only significant variable affecting the BI and TCT outcomes was the treatment type, while the other variables (including the age difference) did not significantly affect the outcomes. It should be noted that $\mathrm{BI}$ and TCT scores were measured completely independently by the physiotherapists, neurologists and orthopaedists; the CAM doctors never took part in these evaluations. It is interesting to note the improvement in the two variables in the IM group in comparison with the group treated with conventional therapy alone, with a significance of $p=0.001$ for TCT and 
$\mathrm{p}<0.001$ for $\mathrm{BI}$. The latter is very important in evaluating stroke and post-stroke rehabilitation, as it analyzes ten variables describing activities of daily living (such as feeding, dressing, grooming, bathing and so on) and mobility (transfer from bed to a chair, walking on a level surface, climbing up and down stairs) $[25,26]$. Clearly, a higher score during the functional recovery phase is associated with a greater chance of being able to live more independently at home after discharge from the rehabilitation facility.

The adverse effects of homeopathy and acupuncture have been monitored over the years, with no adverse events documented with homeopathic magistral preparations; minor adverse events were reported with acupuncture treatments, such as transient bleeding, mild hematoma, and slight pain at the acupoint. No serious adverse events have been reported.

The use of IM in this facility also has other benefits, including the possibility of integrated treatment of comorbidities such as dyslipidaemia and atherosclerosis and help in giving up smoking, all of which are important factors for the reduction of recurrences [27]. CAM doctors spend enough time with patients to convey their more natural outlook on life, and are particularly careful to support the patients' healthier lifestyle choices. Treatment of comorbidities also involves CAM experts beyond the duration of the patients' hospitalization, in cases where they receive follow-up at the IM center's outpatient clinics.

In regards to the use of CAM in stroke patients [28-32], our case showed that CAM is also used by people aged 65 and over without a high level of education, in contrast with literature reports [33]. This is due to its availability in a public health facility, thus bringing it within the reach of people who were not previously aware of it, but who choose to make use of it in any case. On the one hand, they trust the facility in which it is offered, and on the other, they are made aware of the benefits of CAM through word of mouth from other patients who have benefited from it, which is easily passed on in such a small facility [7]. It is now certain that acupuncture offers benefits to stroke patients, as also reported in a 2010 review [17, 28], whereas the benefits of homeopathy have not been unambiguously confirmed $[12,22,23]$. To our knowledge, there are no reports in the literature of experiences with an IM protocol similar to that described herein, namely homeopathy plus acupuncture. Naturally, we are unable to say if one is more effective than the other, as we have always used them both together. However, the objective of this study was not to find out if one form of CAM is more effective than another, but to establish if IM is more effective than conventional treatment alone in the management of patients with severe disabilities - a hypothesis that has proven true. It should also be noted that the costs for this type of treatment are very modest, at no more than seven euros per patient per week [7].

\section{Conclusions}

A clinical setting of $\mathrm{IM}$ in a public neurological rehabilitation facility proved effective in improving the rehabilitation performance of post-stroke inpatients in comparison with patients treated with conventional techniques alone. We hope that these results will encourage other public health facilities to embrace the use of $I M$, as it can be effective and safe and have economical choice associated with high patient satisfaction. 


\section{Acknowledgments}

The authors wish to thank all medical staff at the regional health service rehabilitation facility at Manciano, Tuscany for their hospitability and active cooperation in managing the IM setting. They also wish to thank the Tuscany Regional Authority and the ASL Sudest for their institutional support over these years. Finally, thanks to SIOMI (Società Italiana di Omeopatia e Medicina Integrata - Italian Society of Homeopathy and Integrated Medicine), for its scientific support during the writing of this manuscript.

\section{Author Contributions}

All Authors equally contributed to the present study. At the time of this paper Francesca Bechini worked as Medical Trainee in the Centre of Integrated Medicine and she has collaborated to the data collection.

\section{Competing Interests}

The authors have declared that no competing interests exist.

\section{References}

1. Spread - Stroke Prevention and Educational Awareness Diffusion Ictus cerebrale: Linee guida italiane di prevenzione e trattamento. VIII edizione - 1997-2017. https://www.osservatorioictusitalia.it/pubblicazioni/spread-ictus-cerebrale-linee-guidaitaliane-prevenzione-trattamento/

2. Truelsen T, Piechowski-Jóźwiak B, Bonita R, Mathers C, Bogousslavsky J, Boysen G. Stroke incidence and prevalence in Europe: A review of available data. Eur J Neurol. 2006; 13: 581598.

3. Andrews K, Brocklehurst JC, Richards B, Laycock PJ. The rate of recovery from stroke - and its measurement. Int Rehabil Med. 1981; 3: 155-161.

4. Ferrucci L, Bandinelli S, Guralnik JM, Lamponi M, Bertini C, Falchini M, et al. Recovery of functional status after stroke. A post rehabilitation follow-up study. Stroke. 1993; 24: 200-205.

5. Musicco M, Emberti L, Nappi G, Caltagirone C. Italian multicenter study on outcomes of the rehabilitation of neurological patients. Early and long-term outcome of rehabilitation in stroke patients: the role of patient characteristics, time of initiation, and duration of interventions. Arch Phys Med Rehabil. 2003; 84: 551-558.

6. Franck JA, Smeets RJEM, Seelen HAM. Changes in arm-hand function and arm-hand skill performance in patients after stroke during and after rehabilitation. PLoS One. 2017; 12: e0179453.

7. Bernardini S, Cracolici F, Ferreri R, Rinaldi M, Pulcri R. Integration between orthodox medicine, homeopathy and acupuncture for inpatients: Three years' experience in the first hospital for Integrated Medicine in Italy. J Tradit Complement Med. 2015; 5: 234-240.

8. Bernardini S, Gottard A, Rinaldi M, Mattei A, Virgili G, Cracolici F, et al. Effect of integrated medicine on physical performances of orthopaedic and stroke patients: A propensity scorematched study. Altern Integr Med. 2017; 6: 255-261. 
9. Shin YI, Yang $\mathrm{CY}$, Joo MC, Lee SG, Kim JH, Lee MS. Patterns of using complementary and alternative medicine by stroke patients at two university hospitals in Korea. Evid Based Complement Alternat Med. 2008; 5: 231-235.

10. Wei YC1, Sun MF, Chang KC, Chang CJ, Hung YC, Lin YJ, et al. Pilot scheme of health policy in stroke adjuvant acupuncture therapy for acute and subacute ischemic stroke in Taiwan. Evid Based Complement Alternat Med. 2011; 2011: 689813.

11. Shah SH, Engelhardt R, Ovbiagele B. Patterns of complementary and alternative medicine use among United States stroke survivors. J Neurol Sci. 2008; 271: 180-185.

12. Yam W, Wilkinson JM. Is acupuncture an acceptable option in stroke rehabilitation? A survey of stroke patients. Complement Ther Med. 2010; 18: 143-149.

13. Zhang C, Wen Y, Fan X, Yang S, Tian G, Zhou X, et al. A microarray study of middle cerebral occlusion rat brain with acupuncture intervention. Evid Based Complement Alternat Med. 2015; 2015: 496932.

14. Zhang JH, Wang D, Liu M. Overview of systematic reviews and meta-analyses of acupuncture for stroke. Neuroepidemiology. 2010; 42: 50-58.

15. Wu P, Mills E, Moher D, Seely D. Acupuncture in poststroke rehabilitation. A systematic review and meta-analysis of randomized trials. Stroke. 2010; 4: e171-e179.

16. Lu L, Zhang XG, Zhong LL, Chen ZX, Li Y, Zheng GQ, et al. Acupuncture for neurogenesis in experimental ischemic stroke: A systematic review and meta-analysis. Sci Rep. 2015; 6: 19521.

17. Yang A, Wu HM, Tang JL, Xu L, Yang M, Liu GJ. Acupuncture for stroke rehabilitation (Review). Cochrane DB Syst Rev. 2016; 8: CD004131.

18. Lee JA, Park SW, Hwang PW, Lim SM, Kook S, Choi KI. Acupuncture for shoulder pain after stroke: A systematic review. J Altern Complement Med. 2012; 18: 818-823.

19. Sun $Y$, Xue SA, Zuo Z. Acupuncture therapy on apoplectic aphasia rehabilitation. J Tradit Chin Med. 2012; 32: 314-321.

20. Xia W, Zhen C, Zhu S, Tang Z. Does the addition of specific acupuncture to standard swallowing training improve outcomes in patients with dysphagia after stroke? A randomized controlled trial. Clin Rehabil. 2016; 30: 237-246.

21. Chapman EH, Weintraub RJ, Milburn MA, Pirozzi TO, Woo E. Homeopathic treatment of mild traumatic brain injury: A randomized, double-blind, placebo-controlled clinical trial. J Head Trauma Rehabil. 1999; 14: 521-542.

22. Bell IR. Adjunctive care with nutritional, herbal, and homeopathic complementary and alternative medicine modalities in stroke treatment and rehabilitation. Top Stroke Rehabil. 2007; 14: 30-39.

23. Jonas $\mathrm{W}$, Lin $\mathrm{Y}$, Williams A, Tortella F, Tuma R. Treatment of experimental stroke with lowdose glutamate and homeopathic Arnica montana. Perfusion. 1999; 12: 452-462.

24. Chen L, Fang J, Ma R, Gu X, Chen L, Li J, et al. Additional effects of acupuncture on early comprehensive rehabilitation in patients with mild to moderate acute ischemic stroke: $A$ multicenter randomized controlled trial. BMC Complement Altern Med. 2016; 16: 226.

25. Wade DT, Collin C. The Barthel activities of daily living index: A standard measure of physical disability? Int Dis Studies. 1987; 10: 64-67.

26. Franchignoni FP, Tesio L, Ricupero C, Martino MT. Trunk control test as an early predictor of stroke rehabilitation outcome. Stroke. 1997; 28: 1382-1385. 
27. Magnusson G, Ballegaard S, Karpatschof B, Nyboe J. Long-term effects of integrated rehabilitation in patients with stroke: A nonrandomized comparative feasibility study. J Altern Complement Med. 2010; 6: 369-374.

28. Wu P, Mills E, Moher D, Seely D. Acupuncture in poststroke rehabilitation. A sistematic review and met-analysis of randomized trial. Holist Nurs Pract. 2010; 41: e171-e179.

29. Pandian JD, Liu M, Misbach J, Venketasubramanian N. Alternative therapies for stroke treatment in Asia. Int J Stroke. 2011; 6: 541-543.

30. Kadir AA, Hamid AH, Mohammad M. Pattern of complementary and alternative medicine use among Malaysian stroke survivors: A hospital-based prospective study. J Tradit Complement Med. 2015; 5: 157-160.

31. Yeh ML, Chiu WL, Wang YJ, Lo C. An investigation of the use of traditional Chinese medicine and complementary and alternative medicine in stroke patients. Holist Nurs Pract. 2017; 31: 400-407.

32. Hsieh CY, Wang SY, Chuang YH, Chen HH. Ischemic stroke patients' decision-making process in their use of western medicine and alternative and complementary medicine. Holist Nurs Pract. 2018; 32: 17-26.

33. Hart J. Poststroke recovery. Emerging complementary therapies. Altern Complemen Ther. 2010; 6: 277-280.

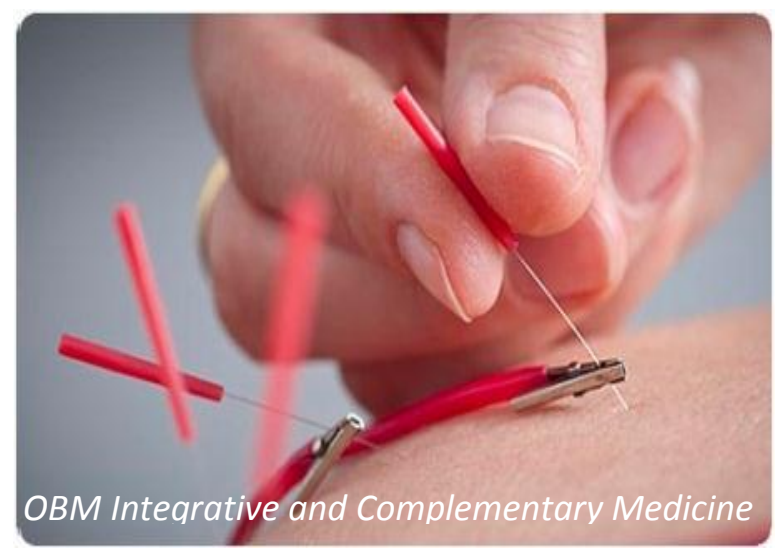

Enjoy OBM Integrative and Complementary Medicine by:

1. Submitting a manuscript

2. Joining in volunteer reviewer bank

3. Joining Editorial Board

4. Guest editing a special issue

For more details, please visit: http://www.lidsen.com/iournals/icm 